BNL-114311-2017-JA

\title{
Local electric field direct writing - electron-beam lithography and mechanism
}

\author{
N. Jiang, D. Su \\ Accepted by the Microelectronic Engineering
}

August 2017

Center for Functional Nanomaterials

Brookhaven National Laboratory

\author{
U.S. Department of Energy \\ USDOE Office of Science (SC), \\ Basic Energy Sciences (BES) (SC-22)
}

\footnotetext{
Notice: This manuscript has been authored by employees of Brookhaven Science Associates, LLC under Contract No. DE-SC0012704 with the U.S. Department of Energy. The publisher by accepting the manuscript for publication acknowledges that the United States Government retains a non-exclusive, paid-up, irrevocable, world-wide license to publish or reproduce the published form of this manuscript, or allow others to do so, for United States Government purposes.
} 


\section{DISCLAIMER}

This report was prepared as an account of work sponsored by an agency of the United States Government. Neither the United States Government nor any agency thereof, nor any of their employees, nor any of their contractors, subcontractors, or their employees, makes any warranty, express or implied, or assumes any legal liability or responsibility for the accuracy, completeness, or any third party's use or the results of such use of any information, apparatus, product, or process disclosed, or represents that its use would not infringe privately owned rights. Reference herein to any specific commercial product, process, or service by trade name, trademark, manufacturer, or otherwise, does not necessarily constitute or imply its endorsement, recommendation, or favoring by the United States Government or any agency thereof or its contractors or subcontractors. The views and opinions of authors expressed herein do not necessarily state or reflect those of the United States Government or any agency thereof. 
Local electric field direct writing - electron-beam lithography and mechanism

Nan Jiang ${ }^{1, *}$, Dong $\mathrm{Su}^{2}$, and John C. H. Spence ${ }^{1}$

${ }^{1}$ Department of Physics, Arizona State University, Tempe, Arizona 85287-1504, USA

${ }^{2}$ Center for Functional Nanomaterials, Brookhaven National Laboratory, Upton, New York, 11973, USA

* Corresponding author: nan.jiang@asu.edu 


\section{ABSTRACT:}

Local electric field induced by a focused electron probe in silicate glass thin films is evaluated by the migration of cations. An extremely strong local electric field can be obtained by the focused electron probe from a scanning electron microscope. As a result, collective atomic displacements occur. This newly revised mechanism provides an efficient tool to write patterned nanostructures directly, and thus overcome the low efficiency of the conventional electron-beam lithography. Applying this technique to silicate glass thin films, as an example, a grid of rods of nanometer dimension can be efficiently produced by rapidly scanning a focused electron probe. This nanopatterning is achieved through swift phase separation in the sample, without any post-development processes. The controlled phase separation is induced by massive displacements of cations (glass modifiers) within the glass-former network, driven by the strong local electric fields. The electric field is induced by accumulated charge within the electron probed region, which is generated by the excitation of atomic electrons by the incident electron. Throughput is much improved compared to other scanning probe techniques. The half-pitch spatial resolution of nanostructure in this particular specimen is $2.5 \mathrm{~nm}$.

KEYWORDS: nano-patterning; composition modulation; local electric field; STEM 


\section{Introduction}

Conventional electron-beam lithography (EBL) creates patterns by scanning a focused electron beam on a surface covered with an electron-sensitive film (e-beam resist) to change the resists' solubility in certain solvents (developers), thereby leaving the resist intact only in the designated areas [1]. Direct-write EBL creates patterns directly on thin films using a focused electron beam without post-irradiation treatment [2]. EBL has the advantage of extremely high spatial resolution (e.g. $\sim 10 \mathrm{~nm}$ ) and the ability to direct writing of arbitrary nanostructures without a mask. However, either conventional or direct-write EBL is a sequential process that exposes a film of resist in a serial fashion. Therefore, the main shortcoming is its low efficiency, which largely limits its current applications to producing photomasks in optical lithography and producing small numbers of nanostructures for research purposes. To overcome this limitation, besides the projection EBL [3] and the use of multi-parallel beams [4], large efforts have also been made to search for suitable resist materials, which are not only able to produce desired nanostructures, but are also extremely susceptible to electron beams and as a result, shorten the exposure time [5].

Electron-beam lithography is the result of electron-beam damage in resist materials. The success for exploring new resists relies on the understanding of damage mechanisms in the materials. Historically, the beam damage has been overwhelmingly interpreted as due to either knock-on displacement or radiolytic

process $[6-8]$. Although two mechanisms operate differently in different materials, they all create Frenkel defects inside specimens and sputter (or desorb) atoms from 
surface. Therefore electron-beam lithography has been considered as the cause of defect accumulation or surface sputtering, and thus the lithography process is electron dose dependent [9]. Under this theoretical framework, the sensitivity of materials to electron beams are measured by the electron dose required to create an individual structure. For example, it was reported that drilling a hole of $5 \mathrm{~nm}$ in diameter in a $250 \mathrm{~nm}$ thick $\mathrm{NaCl}$ film required an exposure dose of $10^{-1} \mathrm{Cm}^{-2}$ [10]. While drilling a similar hole in an $80 \mathrm{~nm}$ thick $\mathrm{AlF}_{3}$ thin film required an exposure dose of $10^{5} \mathrm{Cm}^{-2}$, and this was accompanied by the formation of $\mathrm{Al}$ in areas adjacent to the irradiated areas [11]. Certainly, the low efficiency of electron-beam lithography is caused by this limitation of dose threshold required to form each individual nanostructure. The key operation conditions were then identified as beam energy and electron dose, and the latter was also used as an indication of the sensitivity of resist [9]. The required dose is in order of $1.0 \mathrm{Cm}^{-2}$ in conventional EBL [5].

However this consideration is not fully accurate. Beam damage induced by high-current-density electron beam in a modern scanning transmission electron microscope (STEM) is not just limited to these mechanisms. In semiconductors and insulators, atom displacements are mainly caused by the mechanism of damage by induced electric field (DIEF) [12, 13]. Two of the unique characteristics of this mechanism are (1) it is dose-rate dependent, and (2) the motion of the displaced atoms is in the manner of association and dissociation, which occurs when positively and negatively charged atoms move toward opposite directions. In addition, it also governs the spatial resolution of EBL, which can be controlled by the strength of the 
induced electric field though manipulating beam current density from an electron probe [14]. The electric field is induced by ejection of secondary and Auger electrons due to electronic excitations and ionizations [12 - 16]. If the induced electric field is strong enough to overcome the activation energy for ion migration, the atoms of the same kind may be displaced collectively $[12,13]$. By this means a type of swarm behavior causes the same species of atoms to move together coherently. Although the theory of this mechanism was not given until in the recent studies $[12,13]$, the experimental phenomena have been widely observed for several decades [17 - 24]. Due to the same reason, the DIEF mechanism has not been seriously considered in EBL. In the previous EBL studies, hole-drillings have been related to the possible involvement of electric field $[15,18]$, but it was not seriously considered until in the recent discussion of spatial resolution limitation of EBL [14].

Although the mechanism of DIEF has been applied to understanding the spatial resolution of EBL [14], the detailed processed and applications have not yet been introduced. In this work, we demonstrate a revived technique for EBL that uses the highly localized electric field induced by a focused electron probe in a STEM. Patterning nanostructures using this technique is achieved through manipulating phase separation, which is triggered by the electric field and occurs rapidly. This electric-field driven lithography is determined by the dose rate (current density of electron beam) rather than the dose. Therefore, the conventional understanding of both efficiency and spatial resolution of electron-beam lithography needs to be updated. It should be pointed out that this technique is currently limited to prototyping 
of research devices.

For convenience, here we use thin films of silicate glasses as examples for demonstration. Micro- and nanolithography in transparent materials are promising approaches for introducing new functionalities into glasses in electronic applications and nanostructures. Previously, most studies were focused on the use of high-power ultra-short (e.g. femtoseconds) pulsed lasers to modify glasses [25-28], since the focused sub-bandgap wavelength fs-laser pulses can efficiently and precisely deposit energy into a micron-sized volume, and induce localized structural and chemical changes inside the bulk, or on the surface [29]. Unfortunately, the spatial resolution of this technique is limited by the wavelength of laser beam, and it is impossible to construct structures on a nanometer scale. In STEM, beam electrons have much shorter wavelength (e.g. wavelength $\lambda=2.5 \mathrm{pm}$ for a $200 \mathrm{keV}$ electron). Although it is difficult to modify bulk glass due to its short penetration distance, electron beam is ideal for nano engineering either self-supported or supported thin film glasses with thicknesses of less than about a micron. Since the physical and optical properties of glass depend on its composition [30], new functionalities of glass can thus be achieved without further chemical etching processes. It should be pointed out that this lithographic technique can be also applied to other materials.

\section{Experimental}

Silicate glass used in this study has nominal composition of $25 \mathrm{CaO}-75 \mathrm{SiO}_{2}$ (mol\%). The glass was made by the conventional melt-and-quench method. The TEM 
specimens were prepared using a standard wedge polish technique from a Multi-prep system (Allied High Tech.). The polished specimens were Ar-ion milled (1.5kV) at liquid nitrogen temperature for five minutes using a Gatan Precision Ion Polishing System (PIPS). A 200kV JOEL-2010F TEM/STEM was used for both nanopatterning and characterization. In STEM mode, a high current density probe (10pA, 0.2-0.5nm in diameter) was used for nanopatterning, and a low current density probe (1.0pA, $0.5 \mathrm{~nm}$ in diameter) was used for characterization. The microanalysis of the nanostructures produced was carried out by electron energy-loss spectroscopy (EELS). EELS spectra were collected using a Gatan Enfina electron spectrometer with energy resolution of $1.0 \mathrm{eV}$. The acquisition time was $10^{-6} \mathrm{~s}$ for each spectrum. Both imaging and electron diffraction were also carried out using TEM mode.

\section{Results}

Figure 1(a) shows a TEM phase-contrast bright-field image of a grating pattern produced by rastering a focused electron probe $(\sim 0.2 \mathrm{~nm}$ in diameter) across the glass. Considering that the TEM image is a $2-d$ projection of a $3-d$ structure along the beam direction (defined as the z-axis here), each bright dot in Fig. 1(a) represents a "nano-rod", whose length is the thickness of the specimen (detailed later). As shown in an enlarged image (Fig. 1b), the diameter of the nano-rod estimated from the phase-contrast image is about $2.5 \mathrm{~nm}$. Each nano-rod was produced by exposure to the focused electron probe for $0.005 \mathrm{~s}$, and the arrays of nano-rods were created by rastering electron probe along the $\mathrm{x}$ - and $\mathrm{y}$-direction subsequently (as indicated in Fig. 
$1 \mathrm{a}$ and $1 \mathrm{~b}$ ) over an area of about $0.36 \mu \mathrm{m}$ by $0.31 \mu \mathrm{m}$. The scan step was $7.4 \mathrm{~nm}$ along $\mathrm{x}$-direction and $6.2 \mathrm{~nm}$ along $\mathrm{y}$-direction. To produce a $50 \times 50$ nano-rod-array, the total exposure time was only about $7.5 \mathrm{~s}$. It should be pointed out that although the exposure for each nano-rod was approximately in order of $100 \mathrm{Cm}^{-2}$, this is not the sensitivity of the resist, which is discussed later.

Modifications of the structure and composition of these nano-rods can be examined by small-angle electron diffraction, since the spacing between the nano-rods is fairly large. Confined by a selected-area aperture, the small-angle electron diffraction pattern from the array of nano-rods in Fig. 1a was obtained, as shown in Fig. 1c. For amorphous materials, the electron diffraction pattern consists of blurred rings, and the diameter of the first ring is related to the average bond length of the material. In silicate glasses, the first ring usually occurs between $5 \sim 10 \mathrm{~nm}^{-1}$ in the diffraction pattern, which is way out of the range in Fig. 1c. The diffraction spots observed in Fig. 1c are formed by the diffracted beams from the periodically arranged nano-rods. As schematically shown in Fig. 2, the arrays can be considered as a 2-d pseudo-crystal of nano-rods, with lattice parameters of $D_{x}$ and $D_{y}$, respectively. The potential seen by the incident electron can be approximately expressed as $\mathrm{V}=\mathrm{V}_{0}+$ $\Delta \mathrm{V}$, in which $\mathrm{V}_{0}$ is the average potential of amorphous matrix and $\Delta \mathrm{V}$ is the perturbation potential due to the nano-rods. Since the nano-rods are periodically arranged, $\Delta \mathrm{V}$ can be expressed according as a Fourier series as,

$$
\Delta V(\mathbf{r})=\sum_{g} \Delta V_{g} \exp (-2 \pi i \mathbf{g} \cdot \mathbf{r})
$$

in which $\mathbf{g}$ are $2-d$ reciprocal vectors defined by the 2- $d$ pseudo-lattice of nano-rods, 
with units of $g_{x}=1 / D_{x}$ and $g_{y}=1 / D_{y}$. According to electron diffraction theory [31], $\Delta \mathrm{V}_{\mathrm{g}}$ is related to the periodic structure of nano-rods via relation:

$$
\Delta V_{g}=\frac{1}{V_{C}} \sum_{j} \Delta f_{j} \exp \left(-2 \pi i \mathbf{g} \cdot \mathbf{r}_{j}\right)
$$

The sum is over all the atoms in one nano-rod, which has volume of $V_{c}=\pi w^{2} t / 4$ (Fig. 2). Since the nano-rods are embedded in the glass matrix, it is reasonable to assume that $\Delta f_{j}=f_{j}-\bar{f}$, in which $f_{j}$ is the electron scattering factor of the $j$ th atom and $\bar{f}$ is the average electron scattering factor of the glass matrix. Therefore, $\Delta f_{j}$ will only cause significant electron diffraction if the composition of nano-rod is very different from the glass matrix.

The strong diffraction observed in Fig. 1c indicates that the modulation in composition by the focused electron probe is significant. Although the phase-contrast TEM image is associated with $\Delta \mathrm{V}$, its relationship with composition is more complicated compared with the Z-contrast STEM high-angle angular dark field (HAADF) images. Figure 3 compares the phase-contrast TEM and Z-contrast STEM HAADF images of $11 \times 11$ arrays of nano-rods, which were produced by the focused electron probe in the glass. The exposure time was $0.1 \mathrm{~s}$ for each nano-rod, and thus total $12.1 \mathrm{~s}$ was used to produce the arrays. It is seen that the bright contrast in TEM becomes dark in the STEM HAADF image. The ADF intensity profile in the inset of Fig. $3 \mathrm{~b}$ clearly shows a drop of intensity in the nano-rods, indicating a decrease of effective mass relative to the glass matrix. It should be pointed out that the nano-rods are not channels of holes, because the ADF intensities of the nano-rods are much higher than the intensity at a hole in the TEM specimen. 
The composition change in the nano-rod can be further confirmed by spatially resolved EELS analysis. To make analysis easier and more accurate, two continuous lines were created in the glass by scanning the focused electron probe $(0.5 \mathrm{~nm}$ in diameter) with a very small step $(0.3 \mathrm{~nm})$. The STEM HAADF image is shown in Fig. 4(a). Drawing a line in the glass is in fact extending a nano-rod in one dimension, and thus can be considered to be creating a nano-wall. As shown in Fig. 4(a), the nano-walls have dimension of $4 \mathrm{~nm}$ wide and $150 \mathrm{~nm}$ long, and the height is about the thickness of the specimen. Two nano-walls were 55nm apart. Two line scans of ADF intensity profiles, as marked in Fig. 4(a), are compared in Fig. 4(b). The two dips in the ADF profile correspond to the dark contrast in the image, indicating lower effective mass. On a closer look, the intensity mounds can be observed at the sides of dips, indicating larger effective mass.

Figure 5(a) is an EELS spectral image, which was acquired by perpendicularly crossing two nano-walls in Fig. 4(a). The image includes the plasmon peak, Ca $\mathrm{M}_{23}$-edge $(\sim 36 \mathrm{eV})$ and $\mathrm{Si} \mathrm{L}_{23}$-edge $(\sim 108 \mathrm{eV})$ only. The white arrows indicate the positions of nano-walls. It is clearly shown that the intensities of the $\mathrm{Ca} \mathrm{M}_{23}$-edge are significantly lower in the nano-walls. Three representative spectra are compared in Fig. 5(b). The intensity of the $\mathrm{Ca} \mathrm{M}_{23}$-edge is high in the mound region (spectrum B), which is higher than in the glass matrix (spectrum C). By contrast, it becomes very low in the nano-wall region (spectrum A). The bump around $45-50 \mathrm{eV}$ is from the plural scattering of the plasmon in the glass, which peaks at around $24.5 \mathrm{eV}$. After extracting the contribution from plural scattering, it is reasonable to consider that the 
nano-wall region is almost Ca-free. By contrast, as shown in the inset of Fig. 5b, the $\mathrm{Si}_{23}$ edges are almost identical in all these regions. Therefore, under the irradiation of a focused electron beam, the $\mathrm{Ca}$ were driven out of the probed region and accumulated aside, while the Si remained. As a result, the nano-rod created by the electron probe has a shell structure, in which the core of the rod is consistent with $\mathrm{SiO}_{2}$, surrounded by a Ca-rich thin shell.

An alternate method to create nanostructures in glasses using a "fast" scanning focused electron probe is schematically shown in Fig. 6. Different from the method used in Fig. 1, the fast scan method allows overlap of electron probe exposure, and the spacing of nanostructures is determined by the spacing between two subsequent scans. This method has been used in Fig. 4 to create nano-walls. The nano-rod structure can be produced by repeating the scan in two intersecting directions. One special case is that two directions are perpendicular to each other as illustrated in Fig. 6. On the crosses of scans, indicated by red areas in Fig. 6, the cations can be largely removed, resulting Ca-less or even $\mathrm{Ca}$-free regions. The removed cations aggregate in the intact regions, indicated by blue areas in Fig. 6. Therefore, a periodic composition modulation at the nanometer scale can be achieved. One example is given in Fig. 7. The shortest exposure available in our current instrument is $10^{-6} \mathrm{~s}$. For such a short exposure under the same beam current density (10pA within $0.5 \mathrm{~nm}$ in diameter), the scanning step needs to be less than the probe size. In this case, the step was about half of probe size, $\sim 0.2 \mathrm{~nm}$. Under these conditions, the estimated total time for making a grating containing $10^{2} \times 10^{2}$ nanostructures within a $1 \mu \mathrm{m} \times 1 \mu \mathrm{m}$ area is about $20 \mathrm{~s}$. 
On a closer look, the detail of the pattern is quite different from that in Fig. 1: the pattern is not composed of individual nano-rods as in Fig. 1. Instead, it consists of two sets of perpendicular nano-walls, and it has the brightest contrast at the intersection of the nano-walls. Nevertheless, small-angle electron diffraction from a selected area of gratings in Fig. 8 shows strong diffraction from the periodic lattices, indicating a significant modification of the glass. As shown in Fig. 7, "defects" can also be created by over-scanning on the existing nanostructures.

We have also observed similar phenomena in other silicate glasses, such as $\mathrm{MgO}-\mathrm{CaO}-\mathrm{SiO}_{2}, \quad \mathrm{GeO}_{2}-\mathrm{SiO}_{2}, \quad \mathrm{Na}_{2} \mathrm{O}-\mathrm{CaO}-\mathrm{SiO}_{2}, \quad$ and $\quad \mathrm{Li}_{2} \mathrm{O}-\mathrm{Nb}_{2} \mathrm{O}_{5}-\mathrm{SiO}_{2}$. Shell-structured nano-rods can be created in all these glasses: the core of the nano-rod is in all cases poor but the shell rich in cations (glass modifiers).

\section{Discussion}

Mechanisms for EBL must be associated with the electron-resist interactions. Generally, there are two types of interactions through elastic and inelastic scattering, respectively [13]. Elastic scattering results in momentum transfer from incident electron to atom, causing atomic displacement if the energy acquired by the atom is high enough to overcome its binding energy (or activation energy for migration). This is the knock-on damage. Inelastic scattering results in electronic excitation and ionization. One of the consequences of the inelastic scattering is the so-called radiolytic damage, in which the decay of electronic excitation breaks chemical bonds and produces atomic displacements. Both knock-on and radiolysis produce 
Frenkel-type defects randomly in the illuminated volume [13]. EBLs from these mechanisms are in principle due to the accumulation of these induced point defects, and thus it is electron dose dependent. However, these cannot account for the lithographic observations in this work. This is because the patterns created by the electron beam are not the accumulation of random point defects, but the consequence of massive coherent atomic displacements: cations $\left(\mathrm{Ca}^{2+}\right)$ are ejected collectively to the surrounding of the electron probe forming a core-shell nanorod structure.

A suggested mechanism is indicated schematically in Fig. 8 [12 - 14]. The excited electrons, either through direct excitations (secondary electrons) or Auger decays, obtain high enough kinetic energy to propagate a distance equal to or longer than its mean-free-path (MFP) before being absorbed or trapped. The MFP can vary from several to hundreds of nanometers, depending on the kinetic energy of the excitation [32]. In STEM, the probe size is usually less than half of one nanometer or even smaller. Therefore, most (if not all) of the excited electrons can propagate out of the probed region, as illustrated in Fig. 8(a). If the resist is electronically insulated, the ejected electrons cannot be compensated immediately by the screening electrons from the adjacent region [12-14]. As a result, the probed region (red in Fig. 8(a)) becomes a positively charged column. An electric field, centering at the probed region and pointing radially outward, can be induced, as schematically shown in Fig. 8(b). Assuming the column is charged uniformly with density $\rho$ (Coulomb per length), the strength of the induced electric field can be simplified as [12]

$$
|\mathbf{E}|=\frac{\rho}{2 \pi \varepsilon_{0} \varepsilon_{r} R}
$$


in which $\varepsilon_{0}$ and $\varepsilon_{\mathrm{r}}$ are the permittivity of free space and the relative permittivity of resist, and $R$ is the shortest distance from the beam position. Thus the induced electric field has an approximate cylindrical symmetry around the probe and its strength is independent of specimen thickness $[12,13,21,23]$. If the induced electric field is so strong that the electric force acting on the atom (ion) can overcome the energy barrier $\mathrm{E}_{\mathrm{a}}$ for atomic migration, this atom can be removed, outward for cation or inward for anion (Fig. 8(b)).

To move a bonded atom in the resist, the work done by the electrostatic force on this atom as it moves from one site to another must be larger than its activation energy, i.e.

$$
\mathrm{W}=\int_{\text {sitel }}^{\text {site } 2} q \mathbf{E}_{\text {ind }}(\mathbf{r}) \cdot \mathbf{d r} \geq E_{a}
$$

in which $q$ is electric charge of the atom. Considering that $\overrightarrow{\mathbf{E}}_{\text {ind }}$ is approximately cylindrical around the electron probe and the distance $(d)$ between the nearest minimum energy sites is small, the minimum work required for the atomic motion can be simplified as

$$
\mathrm{W}_{\min }=d \cdot q \cdot\left|E_{\text {ind }}^{T h}\right|=E_{a}
$$

Therefore, there is a threshold electric field that the lithographic process takes place only if the induced electric field is stronger than $\mathbf{E}_{\text {ind }}^{\text {Th }}$. According to previous studies, the strength of the induced electric field is determined by the current density, i.e. electron dose rate. Therefore, unlike other proposed mechanisms and EBLs, the EBL through the local electric field is dose rate dependent [14].

Obviously, this interaction is more efficient for ionic than covalent bonds, and 
thus in the $\mathrm{CaO}-\mathrm{SiO}_{2}$ glass $\mathrm{Ca}$ is more affected than $\mathrm{Si}$, since the former is ionically but the latter is covalently bound to $\mathrm{O}$. As illustrated in Fig. $8, \mathrm{Ca}^{2+}$ ions are ejected from the region, where the induced electric field has higher potential than the activation energy $\mathrm{E}_{\mathrm{a}}\left(\mathrm{Ca}^{2+}\right)$ for $\mathrm{Ca}^{2+}$ migration, forming a core region of depleted or absent $\mathrm{Ca}$. The ejected $\mathrm{Ca}^{2+}$ ions accumulate around the core, forming a thin Ca-rich shell. Accordingly, the lateral size of the nano-rod depends on the strength of the induced electric field, which is determined by the current density of the electron probe and exposure time [14]. The length of the nano-rod is the thickness of the specimen.

Considering that the probe size is very small $(\sim 0.5 \mathrm{~nm}$ in diameter $)$ and the strength of electric field as created by the charged column is inversely related to the distance from the probed region [14], the induced electric field can be very localized. Therefore, moving a focused electron probe in glass is equivalent to moving a localized positive electric filed. The distribution of ions can then be manipulated by moving the focused electron probe correspondingly. Therefore, we can consider the focused STEM probe as an "electric-field pen", by which various patterns can be drawn in silicate glasses. In principle, this process can be very efficient because atom movement under the electric field is massive and simultaneous, opposite to the knock-on and radiolysis process. The produced nanostructure passes through the whole specimen thickness, and thus can be considered as a 3D structure $[11,13,24$, 25]. In other words, there is no request for specimen thickness as long as the energetic electron beam can pass through the specimen, which can be as thin as a few nanometers to as thick as several microns. In addition, specimen composition is not 
crucial either, as long as the specimen is insulated to electrons, which allows charge accumulation within the probed column, and containing weakly bonded cations/anions, which gives rise to low threshold electric fields for lithography.

As we knew, the development of EBL was motivated by pursuing high spatial resolution. Although the electron beam can be focused as small as one tenth of a nanometer in modern electron microscopes, the previous knowledge explained that the spatial resolution of EBL is not set by electron optics, but was determined by the interaction range of the electrons with the resist [33]. The considered interaction includes the straggling of secondary electrons into the resist [34] and the Coulomb interaction between the electrons and the resist atoms [35]. In the former, the average MFPs of the secondary electrons determines the resolution. The latter is similar to the phenomenon of aloof, in which electrons in the beam can interact with specimen although they do not enter, but pass close to it [36]. These considerations, however, do not affect spatial resolution of EBL by the local electric field [14]. Instead, the resolution is determined by the beam current density and the property of the resist. The higher the current density, the stronger the induced electric field, and thus the higher throughput efficiency but lower spatial resolution. In theory, this technique should be able to achieve spatial resolution as good as the size of the electron probe, i.e. set by electron optics, if the induced electric field is just above the threshold strength [14]. Attempts has been made to create smaller conducting nano-channels in insulating $\mathrm{Li}_{4} \mathrm{Ti}_{5} \mathrm{O}_{12}$ battery anode materials, in which an array of slightly over $1 \mathrm{~nm}$ conducting nano-channels with $2.5 \mathrm{~nm}$ spacing has been created using this technique 


\section{Conclusions}

In summary, we have demonstrated a novel EBL technique to modify insulating resists by using the focused electron probe in a STEM instrument. The driving force for nano-modulation is the localized electric field, which is induced by excitations and ionizations of the incident electrons. Under the interaction of the localized electric field, cations in the resists can be relocated efficiently. A periodic array of nano-rods or nano-walls can be produced with very high throughput without a post-development process, and the functionality modification has been examined by small-angle electron diffraction. The direct-write nano fabrication approach described in this paper has potential applications in nano-patterning of insulating materials for various nano-structured devices.

\section{Acknowledgements:}

This work was supported by DOE award DE-FG03-02ER45996. The work of D. S. is supported by Center for Functional Nanomaterials, Brookhaven National Laboratory, which is supported by the U.S. DOE, Office of Basic Energy Sciences, under Contract No. DE-AC02-98CH10886.We are grateful to Dr. J. Qiu for providing the silicate glasses samples, Dr. G. Zhao for helping in Digital Micrograph scripting. 


\section{References}

[1] Xia, Y.; Rogers, J. A.; Paul, K. E.; Whitesides, G. M. Unconventional Methods for Fabricating and Patterning Nanostructures. Chem. Rev. 1999, 99, 1823-1848.

[2] Ito, Y.; Bleloch, L.; Brown, L. M. Nanofabrication of solid- state Fresnel lenses for electron optics. Nature 1998, 394, 49-52.

[3] J. M. Gibson and S. D. Berger, Appl. Phys. Lett., 57, 153 (1990).

[4] E. Slot, M. J. Wieland, G. de Boer, P. Kruit, G.F. ten Berge, A. M. C. Houkes, R. Jager, T. van de Peut, J. J. M. Peijster, S. W. H. K. Steenbrink, T. F. Teepen, A. H. V. van Veen, and B.J. Kampherbeek. MAPPER: high throughout maskless lithography. Proc. of SPIE Vol. 6921, 69211P, (2008).

[5] Chen, Y. Nanofabrication by electron beam lithography and its applications: a review. Microelectronic Engineering 2015, 135, 57-72.

[6] L. Reimer, Transmission Electron Microscopy: Physics of Image Formation and Microanalysis, $2^{\text {nd }}$ ed., (Springer-Verlag Berlin Heidelberg 1989), pp.

[7] D. B. Williams and C. Barry, Transmission Electron Microscopy: A Textbook for Materials Science, (Plenum Press, New York, 1996).

[8] Saifullah, M. S. M. Sub-10 nm direct patterning of oxides using an electron beam - a review. COSMOS 2009, 5, 1-21.

[9] M. A. Mohammad, M. Muhammad, S. K. Dew, and M. Stepanova, Fundamentals of electron beam exposure and development, in Nanofabrication Techniques and Principles, edited by M. Stepanova and S. Dew (Springer 2012), pp. 11-41.

[10] Broers AN, Cuomo J, Harper J, Molzen W, Laibowitz R and Promerantz M, High resolution electron beam fabrication using STEM, in J. M. Sturgess (Ed.), Proc. $9^{\text {th }}$ Int. Cong. Electron Microscopy, Toronto, Ontario, Vol. 3, pp. 343-354, 1978.

[11] Muray A, Isaacson $\mathrm{M}$ and Adesida $\mathrm{I}, \mathrm{AlF}_{3}$ - A new very high resolution electron 
beam resist, Appl. Phys. Lett. 45:589-591, 1984.

[12] Jiang, N. Damage mechanisms in electron microscopy of insulating materials. J, Phys. D: Appl. Phys. 46, 305502 2013. .

[13] Jiang, N. Electron beam damage in oxides: a review. Reports on Progress in Physics 79 (2016) 016501.

[14] N. Jiang. On the spatial resolution limit of direct-write electron beam lithography. Microelectronic Engineering 168 (2017) 41-44.

[15] C. J. Humphreys, T. J. Bullough, R. W. Devenish, D. W. Maher, P. S. Turner, Electron beam nano-etching in oxides, fluorides, metals and semiconductors. Scanning Electron Microscopy Supplement 4, 185-192 (1990).

[16] Cazaux, J. Correlations between ionization radiation damage and charging effects in transmission electron microscopy. Ultramicroscopy 1995, 60, 411-425.

[17] DeNatale; J. F. and Howitt, D. G. A mechanism for radiation damage in silicate glasses. Nucl. Instr. Meth. B 1984, 1, 489-497.

[18] S. D. Berger, I. G. Salisbury, R. H. Milne, D. Imeson, and C. J. Humphreys, Electron energy-loss spectroscopy studies of nanometre-scale structures in alumina produced by intense electron-beam irradiation. Phil. Mag. B 55 (1987) 341-358.

[19] D. Bouchet and C. Colliex, Experimental study of ELNES at grain boundaries in alumina: intergranular radiation damage effects on Al-L23 and O-K edges. Ultramicroscopy 96 (2003) 139.

[20] Jiang N.; Silcox, J. Electron irradiation induced phase decomposition in alkaline earth multi-component oxide glass. J. Appl. Phys, 2002, 92, 2310-2316.

[21] Jiang, N.; Qiu, J.; Garta, A.; Silcox, J. Nanoscale modification of optical properties in Ge-doped $\mathrm{SiO}_{2}$ glass by electron-beam irradiation. Appl. Phys. Lett. 
2002, 80, 2005.

[22] Jiang, N.; Qiu, J.; Ellison, A.; Silcox, J. Fundamentals of high-energy electron-irradiation-induced modifications of silicate glasses. Phys. Rev. B 2003, $68,064207$.

[23] Jiang, N.; Humbree, G.; Spence, J. C. H.; Qiu, J.; de Abajo, F. J. G.; Silcox, J. Nanoring formation by direct-write inorganic electron-beam lithography. Appl. Phys. Lett. 2003, 83, 551-553.

[24] Mkhoyan, K. A.; Silcox, J.; Ellison, A.; Ast, D.; Dieckmann, R. Full Recovery of Electron Damage in Glass at Ambient Temperatures. Phys. Rev. Lett. 2006, 96, 205506.

[25] Davis, K. M.; Miura, K.; Sugimoto, N.; Hirao, K. Writing waveguides in glass with a femtosecond laser. Opt. Lett. 1996, 21, 1729-1731.

[26] Glazer, E. N.; Milosavljevic, M.; Huang, L.; Finlay, J. R.; Her, T. -H.; Callan, J. P.; Mazur, E. Three-dimensional optical storage inside transparent materials. Opt. Lett. 1996, 21, 2023-2025.

[27] Qiu, J.; Jiang, X.; Zhu, C.; Shirai, M.; Si, J.; Jiang, N.; Hirao, K. Manipulation of Gold Nanoparticles inside Transparent Materials. Angew. Chem. Int. Ed. 2004, 43, 2230-2234.

[28] Su, D.; Jiang, N.; Qiu, J.; Spence, J. C. H. Microstructures induced by femtosecond laser pulses inside glasses. J. Mater. Res. 2009, 24, 1983-1988.

[29] Shimotsuma, Y.; Hirao, K.; Kazansky, P. G.; Qiu, J. Three-Dimensional Microand Nano-Fabrication in Transparent Materials by Femtosecond Laser. Jpn. Appl. Phys. 2004, 44, 4735-4748.

[30] Weber M. Optical properties of glasses. In Materials Science and Technology: Glasses and Amorphous Materials, vol. 9, Zarzycki, J., Ed.; VCH Publishers Inc.: 
New York, 1991; pp 619-644.

[31] Spence, J. C. H.; Zuo, J. M. Electron Microdiffraction; Plenum Press: New York, 1992.

[32] Seah M. P.; Dench, W. A. Quantitative electron spectroscopy of surfaces: A standard data base for electron inelastic mean free paths in solids. Surf. Interface Anal. 1979, 1, 2.

[33] A. N. Broers, A. C. F. Hoole, and.M. Ryan. Electron beam lithography resolution limits. Microelectronic Eng. 32 (1996) $131-142$.

[34] D. C. Joy. The spatial resolution limit of electron lithography. Microelectronic Eng. 1 (1983) $103-119$.

[35] M. I. Lutwyche. The resolution of electron beam lithography. Microelectronic Eng. 17 (1992) $17-20$.

[36] A. Howie and R. H. Miline. Electron energy loss spectra and reflection images from surfaces. J. Microscopy 136 (1984) 279-285.

[37] Su. D.; Wang, F.; Ma, C.; Jiang, N. Engineering nano-composite $\mathrm{Li}_{4} \mathrm{Ti}_{5} \mathrm{O}_{12}$ anodes via scanning electron-probe fabrication. Nano Energy 2, 343 (2013). 


\section{Captions}

Figure 1 (a) TEM phase-contrast image of a produced nanostructure using a focused scanning electron probe. (b) Enlarged and rotated image showing the details of produced "lattices". (c) Small-angle electron diffraction from the produced nanostructure.

Figure 2 Schematic drawing of nano-rods formed in the glass by scanning a focused electron probe. $\mathrm{D}_{\mathrm{x}}$ and $\mathrm{D}_{\mathrm{y}}$ are spacing distances along the $\mathrm{x}$ - and $\mathrm{y}$-direction, respectively, $w$ is diameter of nano-rod, and $t$ is specimen thickness.

Figure 3 TEM phase-contrast image $\{$ in (a) $\}$ and STEM HAADF image $\{$ in (b) $\}$ of the same nano-rods pattern produced in the glass. The inset is the ADF intensity profile along the white line. The black arrows indicate the positions of nano-rods, i.e. the black dots, and "hole" refers to vacuum.

Figure 4 (a) STEM HAADF image showing two nano-walls formed in the glass. (b) Comparison of ADF intensity profiles across nano-walls with the profile from the matrix glass. Two lines in (a) indicate the positions where the intensity profiles were obtained.

Figure 5 (a) EELS spectra image recorded across the nano-walls. Two white arrows indicate the position of nano-walls. (b) Comparison of the selected background-subtracted EELS spectra. The upper case letters indicate the locations of spectra in Fig. $4 \mathrm{~b}$.

Figure 6 (a) Schematic projection showing overlapped scanning probe along $\mathrm{x}$ - and y-direction, respectively. The red regions represent $\mathrm{Ca}$-less or $\mathrm{Ca}-\mathrm{free}$, while the 
light blue regions represent Ca-rich region. (b) is the 3-d version of (a).

Figure 7 (a) TEM phase-contrast image of a produced nanostructure by scanning an overlapped electron probe along $\mathrm{x}$ - and $\mathrm{y}$-direction, respectively. The steps for $\mathrm{x}$ and $y$ scans are $\Delta x=6.5 \mathrm{~nm}$ and $\Delta y=8.5 \mathrm{~nm}$, respectively. The inset is the small-angle electron diffraction from the grating area. (b) Enlarged and rotated image showing the details of lattices and defect.

Figure 8 Schematic drawing showing the mechanism for the formation of nano-rod by the focused electron probe $\{$ in (a) $\}$, electric field potential distribution $\{$ in (b) $\}$, and $\mathrm{Ca}$ distribution $\{$ in (c) $\}$. The signs of the circled $\mathrm{c}$ represent cations. $\mathrm{E}_{\mathrm{a}}\left(\mathrm{Ca}^{2+}\right)$ denotes the activation energy for $\mathrm{Ca}^{2+}$ migration. 
